\title{
Le mal et la sorcellerie selon George Gifford
}

\author{
Richard Marienstras
}

\section{(2) OpenEdition \\ Journals}

\section{Édition électronique}

URL : http://journals.openedition.org/shakespeare/1150

DOI : 10.4000/shakespeare.1150

ISSN : 2271-6424

Éditeur

Société Française Shakespeare

Édition imprimée

Date de publication : 1 novembre 1997

Pagination : 139-150

Référence électronique

Richard Marienstras, "Le mal et la sorcellerie selon George Gifford ", Actes des congrès de la Société française Shakespeare [En ligne], 15 | 1997, mis en ligne le 01 janvier 2007, consulté le 02 mai 2019. URL : http://journals.openedition.org/shakespeare/1150; DOI : 10.4000/shakespeare.1150 


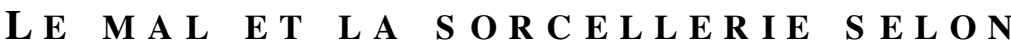 G E O R G E G I F F O R D}

George Gifford, après des études à Hart Hall, Oxford, avant 1568, fut prédicateur à Maldon, dans l'Essex, entre 1582 et 1584, date à laquelle il en fut chassé pour non-conformité. Il est l'auteur d'un nombre assez important de sermons, d'ouvrages théologiques et de textes polémiques contre Henry Barrow et John Greenwood (des brownistes, ou donatistes), tous deux pendus à Tyburn en 1593 pour avoir publié des ouvrages séditieux.

Il est également l'auteur de deux petits livres relatifs à la sorcellerie, dans lesquels il s'efforce de situer clairement l'origine du mal dont les sorciers, les sorcières et les désenvoûteurs, hommes ou femmes, se rendent coupables.

Le premier de ces ouvrages, A Discourse of the subtill Pracices of Deuills by Witches and Sorcerers. By which men are and haue bin greatly deluded : the antiquitie of them : their diuers sorts and Names, a été publié en 1587, soit trois ans seulement après The Discoverie of Witchcraft (1584), le célèbre traité dans lequel Reginald Scot nie l'existence de la sorcellerie. Dans une épître dédicatoire «to the right woorshipfull Maister Richard Martin, Alderman, and Warden of her Maiesties Mint», George Gifford expose les raisons qui lui font écrire ce petit opuscule in quarto de soixante-six pages Les Écritures, dit-il, mentionnent souvent l'existence de sorciers, de conjurateurs, de magiciens («Witches, Coniurers, \& Sorcerers») mais le vulgaire ne comprend que rarement ce que ceux-ci sont véritablement capables de faire (Sig. A2). De plus, «some few are of the minde, that whatsoever is spoken in the holy Scriptures, or in other writers concerning things wrought by such kinde of people, it was not done by the deuill, but was a meere craft and cosenage of the deceiptfull men and women, which have taken vpon them 
such matters» (Sig. A2). Cette fausse perception de l'origine du mal indigne Gifford, puisqu'elle contredit, nous dit-il, le témoignage même de la parole de Dieu. Mais il y a pire encore : «The vain conceit of the multitude... which imagine that Witches can work at their pleasure, and so are the comon plague of the earth, breedeth so innumerable sins, that it is a monster with many heads» (Sig. A2).

Ce qui ressort clairement de cette introduction, c'est que le mal, ou les maux attribués aux sorcières, existent bel et bien et ne sont pas des tours de passe-passe ou des jeux d'illusionnistes. Mais l'erreur impardonnable, c'est de ne pas désigner le diable comme l'auteur de ces maux, et d'en attribuer la responsabilité aux sorcières. Une comparaison avec les idées de Reginald Scot (1538 ?-1599), membre du parlement pour New Romney, montrera bien la différence d'appréciation qu'il y a entre celui-ci et le prédicateur dissident. (Par par une curieuse coïncidence, Reginald Scot fut également étudiant à Hart Hall, Oxford).

Scot, donc, estime que bien des méfaits attribués aux sorcières ne sont que des supercheries: "Sometimes observers of dreames, sometimes soothsaiers, sometimes the observers of the flieng of foules [fowls], of the meeting of todes, the falling of salt, \&c : are called witches. Sometimes he or she is called a witch, that take upon them, either for gaine or glorie, to doo miracles; and yet can doo nothing. Sometimes they are called witches in common speech, that are old, lame, curst, or melancholike, as a nickname. But as for our old women, that are said to hurt children with their eies, or lambs with their lookes, or that pull downe the moone out of heaven, or make so foolish a bargain, or doo such homage to the divell; you shall not read in the bible of any such witches, or of any such actions imputed to them» (p.62-63- V.9) ${ }^{1}$.

Pour revenir au premier ouvrage de Gifford, qui ne présente pas une très grande originalité, l'auteur s'y applique à prémunir ses lecteurs contre les diaboliques astuces de Satan. Il commence par définir la sorcière (ou le sorcier) - «witch» pouvant être masculin ou féminin - comme un individu «that woorketh by the Deuill, or by some deuelish or curious art, either hurting or healing, revealing things secrete, or foretelling things to come, which the deuil hath deuised to entangle and snare mens soules withal unto damnation. The coniurer, the enchaunter, the sorcerer, the deuiner, and whatsoeuer other sort there is, are indeed compassed within this circle» (sig. Bii) ${ }^{2}$

Le parti pris de Gifford apparaît dans les termes mêmes qu'il emploie : d'abord, l'envoûteur se fait un instrument du Diable («one that woorketh by the Deuill»), en agissant par des moyens que le Diable a élaborés pour conduire les âmes à la damnation. Et peu importe que les moyens utilisés heurtent ou guérissent. Le bien, venant du Diable, n'a donc que l'apparence du bien. Mais le Diable, sans la juste colère de Dieu contre les péchés des 
hommes, ne saurait faire le moindre mal. C'est Dieu qui a endurci le cœur des nations profanes et qui a permis au Diable de les séduire, de les jeter dans un précipice de péchés abominables, afin que leur expiation soit des plus terribles.

Le troisième chapitre est consacré à l'énumération des huit sortes de sorciers mentionnés dans le chapitre 18 du Deutéronome (charmeur de charmes, conjectureur, devin, sorcier, noueur de nœud, questionneur de nécromant et augure, consulteur de morts). C'est un chapitre fort long. Gifford s'y livre à de savantes conjectures à propos de chacun des termes hébreux. Là encore, Reginald Scot, pour sa part, se contente de parler de quatre sortes de sorciers, et de passer très rapidement sur l'énumération du Deutéronome (VI.2).

Le quatrième chapitre de Gifford est un petit traité de démonologie historique, et dans le cinquième, il développe l'idée fort orthodoxe selon laquelle les démons n'ont le pouvoir de nuire aux hommes ou aux biens que dans la mesure où Dieu leur en donne licence. Notre auteur prend ensuite position sur cette question, très controversée à l'époque : le Diable peut-il endosser une forme corporelle ? Des citations de l'Ancien et du Nouveau Testament montrent à l'évidence qu'il convient de répondre à cette question par l'affirmative. Il peut également parler aux hommes. Et Gifford consacre de longues pages à réfuter les «frivoles raisons» utilisées par ceux qui pensent le contraire (sig. E-Fv). Au chapitre 8, Gifford en arrive à l'un des points essentiels de son discours : «It is the common opinion among the blind ignorant people, that the cause and the procuring of harme by witchcraft, proceedeth from the Witch, \& that either the Deuill could or would do nothing unlesse he were sent by her. How absurd this conceit is... The Deuill allureth and seduceth men to become Witches, Coniurors, or Enchaunters, he seemeth to be a seruant unto the Witch, but she is his seruant. The coniurors suppose that they bind him by the power of coniuration in which they reckon up the names of God, but he [i.e. the devil] is voluntarily bound, or doth indeed but faine himself to be bound, for shall we think that he would deuise and teach an art whereby he should indeed be bound... ?» (sig. Fvii).

Et le Diable a de bonnes raisons d'agir ainsi, car à faire le mal par le moyen des sorcières, il mène le monde vers d'horribles péchés et se saisit de l'âme des hommes qu'il voue à une damnation éternelle.

Mais le Diable est rusé, il n'apparaît pas en personne. Gifford analyse non sans ingéniosité le comportement de la vieille femme qui s'en va mendier, se fait rabrouer brutalement et maudit celui ou celle qui la rabroue. Elle se tourne vers ses animaux familiers - ses familiers - et leur demande de tourmenter les auteurs du refus. Et cela se produit. L'homme ou la femme au cœur dur sont pris de tourments affreux. Parfois ils meurent. Et que se passe-t-il alors? Une rumeur s'élève contre la sorcière, on l'arrête, on la 
questionne et, à la longue, elle avoue. Oui, elle avoue ce qu'elle a fait : elle raconte sa colère, sa malédiction, l'ordre donné à ses familiers. On la condamne, parfois à mort.

Comme Gifford se gausse alors de tout ce scénario ! Oui, cette mise en scène a été agencée par le Diable, auquel Dieu avait préalablement donné licence d'endurcir le cœur des villageois qui ont refusé l'aumône. Et il a usé de la permission de Dieu pour tourmenter le corps de ces hommes et de ces femmes au cœur dur. Ou pour faire mourir leur bétail. Ou simplement pour prévoir ce qui adviendrait. Et la vieille sorcière, qu'il a incitée à lancer ses malédictions, croit en son âme et conscience qu'elle est la cause de ces événements. Gifford, qui devient alors très éloquent, déplore que de telles séries d'événements puissent se produire en terre chrétienne. Les hommes, au lieu d'élever leurs regards vers Dieu, ne regardent pas plus loin que la sorcière. Ils oublient leurs propres péchés pour ne s'en prendre qu'à la sorcière.

Alors, au chapitre 10 de son discours, Gifford introduit des nuances remarquables, par lesquelles il s'éloigne beaucoup de son contemporain Reginald Scot. Certes, dit-il, les sorcières, les conjurateurs, les enchanteurs, les envoûteurs sont la cause de monstrueuses erreurs, de nombreux blasphèmes lancés contre le saint nom de Dieu, et par conséquent ils méritent de mourir. Mais cela étant dit, nous pouvons maintenant voir les grossières erreurs de la multitude en de telles affaires.

«For they hold that witches should be put to death, and not onely that, but are inflamed with a wonderfull rage and fury to have it accomplished. I call it rage \& not zeale, because it is not to a right end. For if they were so zealous of it for God's honor it were a merueilous good thing : but indeed it is not for any zeale of God that they be so forward, but for an other consideration. Thieues and murtherers, say they, be put to common deaths : how much more sharp deaths \& torments are witches worthy to have laid upon them ? But aske them why : Oh say they, the witches send their spirits and kill and lame men : they kill young infantes : they kill cattle, they raise winds \& tempests, they be the very plagues of men. Here is a false cause, for where doo we euer finde in the holy scriptures, that Witches or Coniurers have such things layd unto their charge ?» (sig Hvi-I).

Et après avoir énuméré les personnes susceptibles de succomber aux ruses du Diable (les coléreux, les mélancoliques...), Gifford conclut que c'est en marchant dans les voies du Seigneur que les hommes trouveront un remède à la sorcellerie et aux maléfices. Ainsi, pour Gifford, l'action omniprésente du Diable n'excuse pas ses agents abusés : ils méritent quand même la mort. Mais il ne faut pas que la populace exige cette mort pour de mauvaises raisons. 
Le second ouvrage qui nous intéresse est $A$ Dialogue concerning Witches and Witchcraft, publié à Londres en 1593 et réédité en 1603. Si certaines idées de l'ouvrage précédent s'y retrouvent, la forme en est entièrement différente, puisqu'il s'agit d'une conversation entre Samuel, sa femme, M.B. le maître d'école, une maîtresse de maison désignée par la lettre R. et enfin Daniel, qui se présente comme un ami de Samuel mais dont on peut penser qu'il est le porte-parole de l'auteur. L'ouvrage, un quarto d'un peu plus de 120 pages, contient une épître dédicatoire à Robert Clarke, juge et baron de l'Échiquier. Dans cette épître, Gifford présente les raisons qui l'ont incité à écrire. Satan, dit-il, est très écouté, on croit ce qu'il dit. Il parle par le truchement des sorciers, des sorcières et des magiciens, et on prête foi. Bien que tous les démons de l'enfer soient enchaînés et bridés par la haute providence de Dieu, au point qu'ils ne peuvent arracher l'aile du moindre petit roitelet sans l'expresse permission du maître de toute la terre, le Diable fait croire aux sorcières qu'à leur demande et pour leur faire plaisir en satisfaisant leurs éclats de colère, leurs esprits malins estropient et tuent des hommes et des bêtes. Ensuite, le Diable les trahit et les pousse à confesser publiquement qu'elles ont commis des méfaits horribles. Ainsi, il dissimule que Dieu donne parfois aux démons le pouvoir d'infliger des tourments physiques aux bêtes et aux hommes. Ces démons agissent comme s'ils faisaient le mal à la demande expresse des sorciers et des sorcières. Ils agissent aussi par l'intermédiaire des dénoueurs et des dénoueuses de sort («cunning men and wise women») ${ }^{3}$.

Gifford, donc, expose dans ce mince traité comment le Diable, avec toute son adresse, agence ces affaires. Le traité a la forme d'un dialogue afin que ses idées soient plus accessibles au vulgaire. Et il est de fait que çà et là, dans ces dialogues, le ton est souvent plaisant et familier. En voici un aperçu.

Alors que Daniel rend visite à Samuel, il le trouve un peu pâle. Serait-il malade? Non, mais de vieilles sorcières mal disposées lui donnent du tourment. Serait-il ensorcelé ? Non, répond Samuel, «mais quand je vais sur mes propres terres, j'ai peur, car de temps en temps je vois un lièvre dont ma conscience me fait comprendre qu'il est une sorcière ou un de ses esprits («spirits») à la façon dont il me fixe. Parfois je vois une hideuse belette couri à travers ma cour, et parfois, dans ma grange, il $\mathrm{y}$ a un grand chat immonde que je n'aime pas du tout» (p. 8) ${ }^{4}$.

Daniel s'inquiète, ou feint de s'inquiéter. Une sorcière lui aurait-elle fait du mal récemment? «Je ne saurais le dire, mais j’ai bien peur que oui. Car il $\mathrm{y}$ en a deux ou trois dans notre ville qui ne me disent rien qui vaille, en particulier une vieille femme. J'ai bien pris soin d'être aussi attentionné avec elle que je l'ai été avec ma propre mère et de lui offrir parfois quelque chose mais je crois que de temps à autre elle me regarde par en dessous. J'avais un cochon qui mangeait sa pâtée avec les autres et qui paraissait aller très bien le soir, et au matin il était raide mort. Certains de mes voisins voudraient que je 
brûle vifs une poule ou un cochon. D'autres me conseillent de demander l'aide d'un désenvoûteur («cunning man») avant que les choses n'empirent» (p.9) ${ }^{5}$.

Naturellement, il y a justement un désenvoûteur à T.B., à vingt miles de distance. Et un voisin de Samuel, vivant à deux miles à peine de sa ferme, avait perdu deux ou trois vaches, six cochons et une jument.

Je poursuivrai maintenant par un passage en anglais, pour faire sentir la tonalité du récit :

«He went to that same man, and told him he suspected an old woman in the parish. And I thinke he told me, that he shewed him her in a glasse, and told him she had three or four impes, some call them puckrels, one like a grey cat, another like a weasel, another like a mouse, a vengeance take them, it is a great pity the country is not rid of them, and told him also what he should do : it is half a yeare ago, and he never had any hurt since. There is also a woman at R.H. five and twenty miles hence, that hath a great name; and great resort there is daily unto her. A neighbour of mine had his child taken lame, a girl of ten yeares old, and such a paine in her backe, that she could not sit upright. He went to that woman, she told him that he had some bad neighbour, the child was forespoken, as she suspected ; marie, if he would go home, and bring her some of the clothes which the child lay in all night, she would tell him certainly. He went home, and put a table napkin about her neck all night, and in the morning tooke it with him, and she told him the child was bewitched indeed, and so told him what he should do, and he had remedie» (p. 9-10).

Mais tout cela ne tire pas Samuel de son embarras, car il ne sait pas s'il vaut mieux aller voir l'homme de T.B. ou la femme de R.H. Il demande conseil à Daniel, lequel répond que pour Samuel, le danger est entre deux chaises. Puis il lui donne enfin son conseil, en l'avertissant qu'il est certainement ensorcelé. Daniel s'épouvante, car il ne ressent rien dans son corps. Mais non, dit l'autre, «le diable a ensorcelé ton esprit en te rendant aveugle et mécréant. Il t'éloigne de Dieu et fait de toi son propre et dévot serviteur en te poussant à chercher de l'aide auprès des démons» (p.11).

Samuel a beau dire qu'il défie le diable, Daniel maintient son point de vue. Samuel semble convaincu, il ne peut tout de même pas se décider à croire que les sorcières n'existent pas. Pourquoi ne pas entrer à la ferme, et discuter avec l'instituteur nommé M.B., qui s'y trouve justement, et qui connaît le latin?

On rencontre d'abord la femme de Samuel, qui se plaint qu'une autre poule est morte dans la nuit et qu'une voisine n'a pas réussi à faire prendre son beurre de toute la semaine : c'est sûrement la faute à une veille femme du voisinage, contre laquelle il faut aller demander de l'aide. 
La scène a été assez adroitement décrite pas Gifford. Le débat dans ce milieu de fermiers a permis de voir comment le mal s'interprétait dans la vie quotidienne. Mais aussi avec quel sérieux certains esprits réagissaient à des superstitions populaires. Dans des études récentes sur la sorcellerie en Angleterre $^{6}$, on a fait remarquer que l'affaiblissement des solidarités villageoises, les enclosures, la disparition des vaines pâtures avaient causé bien des misères, en particulier parmi les vieilles personnes, et surtout les vieilles femmes, auxquelles on refusait de plus en plus souvent l'aumône et vis-à-vis desquelles se développait une mauvaise conscience qui se transformait souvent en haine accusatrice. D'où la floraison de plaintes en sorcellerie au début du XVIIe siècle.

Le maître d'école reprend la discussion à un niveau qui se veut plus élevé que celui où se plaçait Samuel. Il accorde à Daniel que les sorcières sont possédées par le Diable. Mais il est téméraire et insensé de prétendre que ceux qui luttent contre les sorcières ne le font pas également contre le démon. «S vous étiez homme d'une quelconque instruction, dit-il, vous verriez que des contraires ne sauraient être réunis en un même objet, en un même temps, en un même lieu, en un même point de vue : allons, comment pourrait-on détester le diable, défier le diable et ses œuvres et cependant le suivre ?» (p. 14).

C'est ici que Gifford se prépare à asséner son argumentation la plus forte, celle qui distingue entre le réel et l'imaginaire. Il explique à l'instituteur que ce qu'il nomme des contraires, c'est-à-dire haïr le diable et le suivre, il pense que les hommes portent cela en eux, comme des réalités, alors qu'en fait, aveugles et infidèles, ils sont ensorcelés et séduits au point de suivre le diable et de faire sa volonté. Et ce n'est pas parce qu'ils n'évoquent que leur imagination abusée que ces choses seront véritablement en eux. «Que dire d'une pauvre mendiante qui affirme qu'elle est reine? N'en est-elle pas pour autant toujours une mendiante, quémandant son pain ? En sera-t-elle débarrassée de sa vermine ?» (p. 15).

L'instituteur, alors, fait observer que seule l'intention des hommes compte aux yeux de Dieu : «Si les hommes sont sincères dans leur haine du diable, et le défient ainsi que ses œuvres, avez-vous le droit de juger leur conscience et de décréter qu'ils ne le défient que dans leur imagination, alors qu'en fait ils le suivent et l'honorent? Dieu n'est-il pas seul juge du cœu humain ?» (p. 15-16).

L'opposition entre les deux hommes semble recouvrir une conception évangélique de la conduite humaine chez l'un, et une conception quasiment militaire où seuls les actes comptent, chez l'autre. Daniel, à qui Gifford entend bien laisser le dernier mot, va tourner la discussion à son avantage, afin que les menées secrètes du démon ne restent pas invisibles.

«Je sais que Dieu seul peut scruter le cœur quand les choses y sont 
cachées secrètement. [...] Ainsi, si un homme professe honnêtement la foi en Jésus Christ, parfaitement en accord avec la parole de Dieu, passant ensuite sa vie à faire de bonnes actions, il serait très pervers qu'on le jugeât hypocrite et pétri d'orgueil. Et pourtant, il est bien possible que le Seigneur, qui discerne les intentions secrètes du cœur, s'aperçoive qu'il est bel et bien un hypocrite. Inversement, quand un homme affirme par ses paroles qu'il défie le Diable et toutes ses œuvres, et que le jugeant à l'aune de la parole de Dieu on s'aperçoive qu'il est circonvenu, aveugle victime des erreurs du Diable, de l'infidélité et des œuvres maléfiques par lesquelles il accomplit sa volonté et l'honore à la place de Dieu, dirons-nous alors que cet homme est bon parce qu'il dit et imagine qu'il défie le Diable et ses œuvres ? Malheur à ceux qui appellent le bien, mal, et le mal, bien» (Esa. v. 20) (p. 16).

Ainsi, par le truchement de Daniel, Gifford reprend un des thèmes qu'il avait abordés dans son ouvrage précédent, celui du démon metteur en scène. Les maléfices qu'il provoque, le Diable les organise en se servant d'individus qui à première vue devraient être insoupçonnables : ceux qui luttent contre les envoûtements ou les petits et grands malheurs inexplicables de la vie rurale. Quoi de plus diabolique, en effet, que de prendre les apparences de la lutte contre le malheur pour entraîner les hommes vers le plus grand malheur possible ? C'est pourquoi Daniel prend la peine de démontrer longuement à l'instituteur que les familiers des sorcières ne sont là que pour déguiser des desseins plus funestes. «Quand ils prennent les formes de misérables vermines comme des chats, souris, crapauds et belettes, c'est par habileté, afin de dissimuler la puissance de la tyrannie et du pouvoir qu'ils exercent sur le cour des méchants... alors qu'ils s'occupent des choses les plus graves, comme d'inciter les tyrans à la persécution et les méchants à attaquer l'Évangile... à susciter la division et la guerre entre rois et royaumes... à semer partout le désordre et la confusion : ils voudraient faire croire qu'ils ne sont occupés qu'à ces bagatelles, faisant en sorte qu'elles préoccupent l'esprit des hommes afin que ceux-ci ne prêtent aucune attention à ce que les démons font ailleurs» (p. 22-23).

L'instituteur, cependant, demande encore à Daniel s'il convient de disculper les sorcières parce que c'est Dieu et non pas elles qui donne son pouvoir au Diable. Les démons tueraient-ils les hommes et le bétail s'ils n'étaient pas envoyés par les sorcières ? Les maux nous frapperaient-ils toujours s'il n'y avait plus de sorcières ? (p. 33).

Daniel répète inlassablement que le pouvoir des démons vient de Dieu seul, mais que cela n'innocente pas les sorcières : «Car le péché qu'elles commettent est de croire que ce sont leurs familiers qui causent les maux à la demande des démons, alors qu'en fait, lorsque le Diable sait que pouvoir lui a été donné de faire le mal, ou encore quand il sait que la mort ou des maladies 
vont frapper un homme ou une bête, il provoque la colère de la sorcière et la pousse à faire de lui son émissaire» (p. 33).

Ainsi, la sorcière ne devra pas être épargnée, car il faut révéler la grande habileté de Satan, qui fait semblant d'être envoyé par les sorcières, alors qu'il l'est par Dieu, vu que personne d'autre que Dieu n'a ce pouvoir (p. 34). Quant aux autres maux qui frappent les hommes, Dieu les leur envoie pour éprouver leur patience et leur foi, et il se sert de Satan pour cela, comme en témoigne l'exemple de Job le vertueux.

Gifford, progressivement, en vient à expliciter et à préciser l'origine du mal dans le monde. Paradoxalement, le mal vient de Dieu, soit qu'il s'agisse d'une épreuve, soit qu'il s'agisse d'une punition. Le diable n'a pas reçu le moindre pouvoir à cause de la sorcière, mais bien à cause de la méchanceté des hommes, lesquels méritent que par les sorcières, il les séduise bien davantage (p. 36). L'exemple de Job prouve que non seulement les bons, mais encore les très bons peuvent en guise d'épreuve être remis entre les mains de Satan afin d'être tourmentés et soumis à la tentation (p. 37).

L'instituteur, cependant, va revenir à la charge, et se faire expliquer comment et pourquoi les désenvoûteurs réussissent à guérir des maladies ou faire cesser des douleurs. Daniel dira longuement que ces désenvoûteurs ne sont que des avatars des démons, qui s'emparent ainsi de la confiance des âmes simples. Mais, dit l'instituteur, «il n'en reste pas moins que les démons portent secours dans de nombreux cas. Que dis-tu du garçon qui guérit quelques jours seulement après avoir écorché la sorcière, alors que ses plaies étaient infiniment pénibles auparavant et ne pouvaient être soignées ? Que penses-tu de ce qui arrive lorsque le beurre ne veut pas prendre ou quand la boisson ne fermente pas dans la barrique ? Que dis-tu du fait que lorsqu'on brûle vif quelque animal vivant, tel qu'un cochon ou une poule, le mal disparaît ?» (p. 69)

Daniel affirme alors que quelques mots suffisent à répondre à toutes ces questions. Là où le diable a le pouvoir de faire le mal aux hommes, ou aux bêtes, ou aux boissons, ou au beurre, il se contente en fait de laisser le champ libre à la nature et de mettre un terme à ses attaques. Il fait cela très volontiers, afin que les hommes le recherchent et deviennent eux-mêmes des sorciers. Et Daniel confirmera que l'Écriture ordonne qu'on ne laisse pas vivre les sorcières (il cite le fameux verset de l'Exode, XXII.18), et cela s'applique aussi bien aux sorciers qu'aux désenvoûteurs (p. 72).

Le maître d'école est alors pris d'une nouvelle inquiétude. La meilleure chose serait de pendre tous ceux ou celles qui seraient suspects de sorcellerie.

Mais les sorcières travaillent si secrètement avec leurs familiers qu'il est rare qu'on puisse les condamner sur le simple témoignage des hommes et que ceux-ci puissent dire avec certitude les avoir vues ou entendues envoyer leurs 
familiers. Et elles avouent rarement, car la puissance du démon sur elle est si grande qu'il ne les laisse pas avouer. Le maître d'école était même persuadé que si l'on pendait toutes les sorcières, leurs familiers n'auraient alors plus personne pour les employer à faire le mal.

Daniel lui demande alors s'il n'avait eu aucune crainte, tous les suspects étant pendus, que quelques innocents ne soient mis à mort. Le maître d'école répond : «Je trouvais remarquablement efficace de condamner à mort tous les suspects, bien que certains fussent innocents ; une telle solution nous assurait qu'aucun ne resterait en vie».

Daniel: «Ne trouvais-tu pas effroyable de répandre ainsi du sang innocent ?»

M.B. : «Si, mais je préférais voir certains d'entre eux condamnés à tort au fait d'en laisser un en vie qui risquerait de tuer et de détruire un grand nombre d'êtres» (p. 74) [...]

Daniel : «Nous pouvons voir dans les Saintes Écritures que répandre du sang innocent est un acte particulièrement horrible aux yeux de Dieu Tout Puissant : et c'est chose bien douloureuse que d'en voir un pays souillé ; et ceci est une des raisons particulières pour lesquelles Satan se sert des sorcières : car il s'efforce de faire paraître suspects de nombreux innocents... C'est ainsi que des jurys entiers se seront rendus coupables d'avoir versé du sang innocent, en condamnant, en prêtant un serment solennel, des gens soupçonnés sur de vaines conjectures, pour des faits imaginaires et illusoires suscités par l'aveuglement et l'infidélité et la crainte de Satan qui règne chez les ignorants» (p. 76).

Daniel parlera encore longuement de la nécessité de rendre une probe justice, malgré les difficultés de la tâche. On ne peut croire, en effet, ni les sorciers, ni les désenvoûteurs, et Satan n'a de cesse qu'il ne fasse accuser des innocents.

Comment un jury identifiera-t-il une sorcière ? Quelle preuve doit-on fournir (p. 100)? Daniel s'exprime alors avec précaution, la question est sérieuse en effet. Voici ses paroles :

«Ces hommes [les membres du jury] sont assermentés dans ces affaires et c'est une question de vie ou de mort quand ils déclarent quelqu'un coupable de sorcellerie. La question étant très grave, il s'ensuit qu'il doit y avoir soit une preuve satisfaisante apportée par des témoins dignes de foi, soit la confession de la sorcière elle-même. Car si le témoignage est susceptible d'être faux, comme on peut s'y attendre de la part de tout ce qui provient des démons, ou encore, s'il n'est fondé que sur des rumeurs et des présomptions, lesquelles ne peuvent être que le produit de l'extrême habileté de Satan, comme c'est presque toujours le cas, comment ce jury saurait-il répondre devant Dieu de ce dont il ne peut jurer être sûr, alors qu'en procédant de la 
sorte il court le risque de condamner un innocent, comme cela arrive si souvent ?» (p. 100).

Et Daniel de conclure sur une pensée très conforme à la pensée juridique anglaise : «Si la personne soupçonnée est vraiment une sorcière et que cependant aucune preuve ne puisse en être donnée, le jury fera preuve de plus de justice en l'acquittant qu'en la condamnant, car en quoi leur serment les autorise-t-il à procéder par conjectures ou encore à conclure alors qu'ils sont dans l'ignorance ?» (p. 101).

Daniel prendra congé de ses interlocuteurs après avoir débattu de quelques cas concrets de jugement de sorcières, et avoir exhorté ses amis à éviter les pièges et les astuces du démon en écoutant la voix du Seigneur.

Le dialogue ne s'arrête pas là, cependant. Gifford laisse la parole au maître d'école et aux deux femmes annoncées dans la liste des personnages pour montrer sans doute qu'il est bien difficile d'en finir avec la superstition dans les campagnes.

$$
* * *
$$

Ainsi, Gifford, après s'être efforcé de montrer que la tromperie ou l'illusion sont les armes favorites du malin, présente les sorcières et les désenvoûteurs comme de coupables victimes de leurs propres faiblesses et des ruses du démon. Il situe le mal à mi-chemin entre une acceptation humaine dont l'acquiescement se fait dans une sorte de brouillard, et une volonté satanique toujours en éveil, guettant les faiblesses humaines et le signe divin qui l'autorisera à frapper.

La position fondamentale de Gifford, cependant, semble être celle-ci : le Diable est puissant, très puissant, et il n'a donc pas besoin de vieilles femmes ou d'agents humains pour perpétrer ses méfaits. Il en fait des instruments, non tant pour accomplir le mal, mais pour augmenter encore le mal dans le monde et le répandre à la façon d'une épidémie. Le grand danger, nous l'avons vu, est que des chrétiens frappés par le mal se tournent vers des désenvoûteurs pour leur demander du secours, et, ce faisant, augmenter l'emprise maléfique sur les hommes. Dieu seul, et non les sorcières, donne au Diable pouvoir de frapper et de tourmenter. La sorcière n'est qu'une complice abusée. Le Diable, l'agent principal.

Mais la Bible a tout à fait raison de demander que les sorcières soient mises à mort : elles sont les ennemies de Dieu, et leurs blasphèmes sont intolérables - blasphèmes qui dérivent autant de leurs actes que de leurs paroles. Même si, comme dans le cas des désenvoûteuses, elles pensent faire le bien et contrer le Malin, en fait elles travaillent pour le Diable et doivent mourir pour cela. Mais cela pose des problèmes juridiques que Gifford ne songe pas à éluder. 
Ainsi, dans ses deux petits ouvrages, mais surtout dans le second, il a retracé le complexe cheminement du mal dans le monde - un mal qui, étrangement, trouve en Dieu son origine, même s'il doit passer par d'étonnantes médiations. Et c'est en Dieu qu'après la punition ou le repentir des malfaisants, le mal enfin s'abolit.

Richard MARIENSTR AS

Professeur Émérite

Université Paris VII - Denis Diderot

N O T E S

${ }^{1}$ The Discoverie of Witchcraft, avec une introduction, par Montague Summers, Dover Publications, New York, 1972.

${ }^{2}$ Mes remerciements à Ian Willison, naguère directeur des Imprimés à la British Library, qui m'a procuré une photocopie de A Discourse of the subtill Practices of Deuilles by Witches and Sorcerers...

${ }^{3}$ Le folklore français possède un certain nombre de mots pour désigner ce que Gifford (et d'autres auteurs anglais occupés par la sorcellerie) appellent «cunning man», «cunning woman», «wise man» ou «wise woman». Parmi les expressions francaises, on peut citer : femmes sages, femmes bonnes, leveuses de sort, désorcelleuses, toucheuses, médeciennes, devinoux, dénoueurs ou dénoueuses, panseurs, désenvoûteur, conjureur (Littré) [qui enlève le juré de mal]. Mes remerciements à Nicole Pellegrin (C.N.R.S.) qui m'a communiqué une partie de ce vocabulaire.

${ }^{4}$ Notons que l'expression «wise woman» pouvait se prendre en mauvaise part: "At this day [1584] it is indifferent tosay in the English tongue 'she is a witch' or 'she is a wise woman'», Reginald Scot, Discoverie..., V.ix ; Keith Thomas, Religion and the Decline of Magic, Weidenfeld and Nicolson, Londres, 1971, p. 436. 
${ }^{5}$ La pagination renvoie à l'édition de 1842 de $A$ dialogue concerning witches and witchcraftes [Showing] how the diuell deceiueth not onely the witches but many other (première édition : 1593). J'utilise d'autre part, avec quelques modifications, la traduction de ce texte établie par Elizabeth Connes, mémoire de maîtrise de littérature britannique, Université Paris VII, Institut d'anglais Charles V, octobre 1975.

Par exemple, Alan Macfarlane, Witchcraft in Tudor and Stuart England, Routledge and Kegan Paul, Londres, 1970. 